\title{
Tacrolimus in pediatric ulcerative colitis: does it have a role?
}

\author{
Seung Kim \\ Department of Pediatrics, Severance Children's Hospital, Yonsei University College of Medicine, Seoul, Korea
}

\section{Article: Tacrolimus for ulcerative colitis in children: a multicenter survey in Japan (Intest Res 2019;17:476-485)}

Inflammatory bowel disease (IBD) is becoming more common both in the Eastern and Western countries, and the increasing pattern is more rapid in children and adolescents than in the adult population. ${ }^{1,2}$ Compared to patient with late onset UC, those with pediatric onset UC tend to show more severe and widespread disease location at diagnosis and a high rate of disease extension during follow-up., ${ }^{3,4}$ In a population based European study, the 5-year colectomy rate was also higher among patients with pediatric onset UC (8.1\%) than among patients with adult onset UC (4.1\%). ${ }^{5}$

As steroids have a variety of side effects, such as growth impairments, pediatricians are often reluctant to use steroids; however paradoxically the proportion of steroid-dependent or refractory colitis is also higher in the pediatric age group than in adults. ${ }^{6}$ Although new effective drugs with various mechanisms have begun to be used in adult UC patients, many of these drugs are not approved for use in children or not covered by insurance in some countries. Hence, there are more restrictions on the use of new effective drugs for pediatric UC patients. In order to overcome these obstacles while managing pediatric IBD patients, it is important to optimize the currently available drugs or therapies. Tacrolimus, an immunomodulatory, which act as a potent inhibitor of helper-T lymphocytes, is known to be effective in adult $\mathrm{UC}^{7}$ and was sug-

Received October 1, 2019. Revised October 2, 2019. Accepted October 3, 2019. Correspondence to Seung Kim, Department of Pediatrics, Severance Children's Hospital, Yonsei University College of Medicine, 50-1 Yonsei-ro, Seodaemun-gu, Seoul 03722, Korea. Tel: +82-2-2228-2054, Fax: +82-2393-9118,E-mail: pedks@yuhs.ac gested as one option of a bridging therapy to thiopurines or vedolizumab for pediatric UC in an evidence-based guideline from European Crohn's and Colitis Organisation and European Society of Pediatric Gastroenterology, Hepatology and Nutrition. ${ }^{8}$ However, studies of pediatric UC patients treated with this drug are scarce and the optimal indication, dosage and treatment duration have not yet been clarified.

In the current issue of the Intestinal Research, Yanagi et al. ${ }^{9}$ conducted a retrospective multicenter survey to evaluate the effectiveness and safety of tacrolimus for induction and maintenance therapy in Japanese children with UC. Data from 67 UC patients under 17 years of age who were treated with tacrolimus were included in this study. Thirty-nine patients were steroid-dependent and 26 were steroid-refractory; 20 had severe colitis, and 43 had moderate colitis. The initial tacrolimus dose was $0.09 \mathrm{mg} / \mathrm{kg} / \mathrm{day}$ (range, $0.05-0.12 \mathrm{mg} / \mathrm{kg} /$ day), the highest blood trough concentration within 2 weeks was 12.4 $\mathrm{ng} / \mathrm{mL}$ (range, 9.2-15.2 ng/mL), and the median tacrolimus treatment duration was 18.1 weeks (range, 10-41.4 weeks). Regarding short-term effects, the clinical remission rate was $47.8 \%$ (32 patients), and the clinical response rate was $37.3 \%$ (25 patients). Ten patients (14.9\%) did not show treatment effect. However, among 57 patients who showed short-term remission or response, only 8 (14\%) achieved long-term (1-year) remission without relapse or surgery. The remaining 49 required additional treatments, such as biologics (22 patients, $33 \%$ ), tacrolimus re-administration (10 patients, 14.9\%), prednisolone (4 patients, 6\%) and colectomy (20 patients, 29.9\%). According to the study, the mean dose of prednisolone was 
reduced from $19.2 \mathrm{mg} /$ day at the onset of tacrolimus administration to $5.7 \mathrm{mg} /$ day at week $8(P<0.001)$, which reveals the steroid-sparing effect of tacrolimus administration. Although adverse events were identified during tacrolimus treatment in 36 patients (53.7\%), most of the adverse events were mild and only 6 patients $(9.0 \%)$ required discontinuation of tacrolimus owing to adverse events (renal impairment, myelosuppression, intestinal infection, and vomiting), which improved after discontinuation of tacrolimus or appropriate treatment.

These results suggest that appropriate use of tacrolimus is relatively safe in patients with pediatric UC and can be effective in a substantial number of patients with steroid-refractory or dependent UC, thus achieving steroid-sparing effects. However, as only a temporary effect is expected, efforts are required to connect subsequent treatments for the maintenance of remission. Further prospective studies are needed to establish the optimized strategy for using tacrolimus in the management of children and adolescent with UC.

\section{FINANCIAL SUPPORT}

The author received no financial support for the research, authorship, and/or publication of this article.

\section{CONFLICT OF INTEREST}

No potential conflict of interest relevant to this article was reported.

\section{AUTHOR CONTRIBUTION}

Writing and approval of final manuscript: Kim S.

\section{ORCID}

Kim S

https://orcid.org/0000-0003-4373-9828

\section{REFERENCES}

1. Kaplan GG, Ng SC. Understanding and preventing the global increase of inflammatory bowel disease. Gastroenterology 2017;152:313-321.

2. Ghione S, Sarter H, Fumery M, et al. Dramatic increase in incidence of ulcerative colitis and Crohn's disease (1988-2011): a population-based study of French adolescents. Am J Gastroenterol 2018;113:265-272.

3. Van Limbergen J, Russell RK, Drummond HE, et al. Definition of phenotypic characteristics of childhood-onset inflammatory bowel disease. Gastroenterology 2008;135:1114-1122.

4. Ruel J, Ruane D, Mehandru S, Gower-Rousseau C, Colombel JF. IBD across the age spectrum: is it the same disease? Nat Rev Gastroenterol Hepatol 2014;11:88-98.

5. Lakatos PL, David G, Pandur T, et al. IBD in the elderly population: results from a population-based study in Western Hungary, 1977-2008. J Crohns Colitis 2011;5:5-13.

6. Ishige T, Tomomasa T, Takebayashi T, et al. Inflammatory bowel disease in children: epidemiological analysis of the nationwide IBD registry in Japan. J Gastroenterol 2010;45:911917.

7. Komaki Y, Komaki F, Ido A, Sakuraba A. Efficacy and safety of tacrolimus therapy for active ulcerative colitis; a systematic review and meta-analysis. J Crohns Colitis 2016;10:484-494.

8. Turner D, Ruemmele FM, Orlanski-Meyer E, et al. Management of paediatric ulcerative colitis, part 1: ambulatory carean evidence-based guideline from European Crohn's and Colitis Organization and European Society of Paediatric Gastroenterology, Hepatology and Nutrition. J Pediatr Gastroenterol Nutr 2018;67:257-291.

9. Yanagi T, Ushijima K, Koga $H$, et al. Tacrolimus for ulcerative colitis in children: a multicenter survey in Japan. Intest Res 2019;17:476-485. 